\title{
HEPATITIS B PATIENT WITH FEVER DUE TO FOCAL INFECTION
}

Assya Krasteva*, Dobriana Panova**, Krasimir Antonov**, Angelina Kisselova*

* Department of Imaging and Oral Diagnostic, Faculty of Dental Medicine,

** Clinic of gastroenterology, "St. Ivan Rilski" University Hospital,

Medical University - Sofia, Bulgaria.

\section{ABSTRACT}

Persistent undiagnosed fever remains a common problem in clinical practice. It is a fact that dental sepsis is one potential cause of persistent fever that can escape detection (Siminoski, K., 1993).

We present a 50 years old woman with chronic hepatitis $\mathrm{B}$, febrile for the past two months (max. $38.6^{\circ} \mathrm{C}$ ) with characteristic of septic fever. She underwent consultations with endocrinologist, rheumatologist, neurologist, gynecologist, pulmonologist and infectionst. All negative for any disease, also negative serology for Lyme disease. She had no data for urine infection. She had negative cultures. The patient was treated with corticosteroids for 30 days with no effect; she had no response to treatment with Gentamycin, Amoxicillin, Azithromycin, Ciprofloxacin, Methronidazole, Meronem, Clindamicin and Nystatin. Only reduction but not normalization, of CRP was observed, the fever remained.

The patient underwent focal dental diagnostic protocol. We remarked potential dental foci - 17, 16, 27, 43, 47 and active temperature zone - submandibular lymph nodes.

The dental focuses were extracted consequently and the patient became afebrile 5 days afterwards. Her liver enzymes, CRP and albumin returned to normal after performance of dental recommendations.

Key words: fever, focal infection, dental sepsis

\section{INTRODUCTION}

Adult patients frequently visit physicians with date of persistent fever and the cause of the fever sometimes cannot be found.

The definition of fever of unknown origin (FUO), as based on a case series of 100 patients, is defined as a temperature higher than $38.3 \mathrm{C}(100.9 \mathrm{~F})$ that lasts for more than three weeks with no obvious source despite appropriate investigation (Roth, A., 2003), and evaluation of at least 3 outpatient visits or 3 days in hospital (Durack, DT.,1991).

The more then 200 reported causes of FUO can be classified into four diagnostic categories: infections, tumours, noninfectious inflammatory diseases and miscellaneous ( Knockaert, DC., 2003) Alan R. Roth summaries the common etiologies of fever of unknown origin (Tabl.1). He include tuberculosis (especially in extrapulmonary sites) and abdominal or pelvic abscesses, subacute bacterial endocarditis, sinusitis, osteomyelitis, dental abscess etc. (Roth, A., 2003).

Tabl. 1. Common Etiologies of Fever of Unknown Origin by Alan R. Roth

\begin{tabular}{|l|l|l|}
\hline Infections & Pelvic abscesses & Osteomyelitis \\
\hline $\begin{array}{l}\text { Tuberculosis (especially } \\
\text { extrapulmonary) }\end{array}$ & Dental abscesses & Sinusitis \\
\hline Abdominal abscesses & Endocarditis & Cytomegalovirus \\
\hline Epstein-Barr virus & Human immunodeficiency virus & Lyme disease \\
\hline Prostatitis & Chronic leukemia & Malignancies \\
\hline Lymphoma & Metastatic cancers & Renal cell carcinoma \\
\hline Colon carcinoma & Hepatoma & Myelodysplastic syndromes \\
\hline Pancreatic carcinoma & Sarcomas & Autoimmune conditions \\
\hline Adult Still's disease & Polymyalgia rheumatica & Temporal arteritis \\
\hline Rheumatoid arthritis & Rheumatoid fever & Inflammatory bowel disease \\
\hline Reiter's syndrome & Systemic lupus erythematosus & Vasculitides \\
\hline Miscellaneous & Drug-induced fever Allopurinol (Zyloprim) & Complications from cirrhosis \\
\hline Factitious fever & $\begin{array}{l}\text { Hepatitis (alcoholic, } \\
\text { granulomatous, or lupoid) }\end{array}$ & Deep venous thrombosis \\
\hline
\end{tabular}


The patients with persistent fever of unknown origin (FUO) often undergo modern diagnostic procedures (serology, viral cultures, computed tomography, magnetic resonance imaging, termovision investigation (infrared thermographic methods etc) with one goal - to identify the cause of the fever, which rarely may be from dental origin.

\section{CASE DETAIL}

We present a 50 years old woman, febrile for the past two months (max. $38.6^{\circ} \mathrm{C}$ ) with characteristic of septic fever. Reporting for moderate pain and weakness in the four limbs. Diagnosed with chronic hepatitis B two weeks after the fever onset. With elevated aminotrasferases, which progressively reached up to 10 times above the upper limit of normal. The patient had HBV-DNA 533000000 copies $/ \mathrm{ml}$. She had normal blood count and hemoglobin, normal bilirubin and INR; no laboratory data for autoimmune liver disease. She had progressive hypoalbuminemia, elevated CRP and prominent liver necrosis with ALT predomination, no liver decompensation. She underwent consultations with endocrinologist, rheumatologist, neurologist, gynecologist, pulmonologist and infectionst. All negative for any disease, also negative serology for Lyme disease. She had no data for urine infection. She had negative cultures. The patient was treated with corticosteroids for 30 days with no effect; she had no response to treatment with Gentamycin, Amoxicillin, Azithromycin, Ciprofloxacin, Methronidazole, Meronem, Clindamicin and Nystatin. Only reduction but not normalization, of CRP was observed, the fever remained.

The patient underwent focal diagnostic protocol which includes:

- Clinical examination - dental status, palpation of lymph nodes, percussion

- X-ray diagnostics - ortopantomography additional method indicating the presence of different source of inflammation.

- Gelen test (electro-skin test) - for detection active or potential $t$ dental foci

- Electroodontodiagnostics- test for vitality of the teeth

- Thermovision research during Flir A360 in 6 six aspects

Thermography is not invasive to the human body and is non-contact technique through which the temperature distribution of the human body surface can be monitored and recorded - physiological asymmetry $\mathrm{t}-0,4$.

\section{RESULTS}

- Swollen lymph nodes

- No vital teeth - № 17, 47,

- X - ray periapical and peridontal changes on 17 , 16, 27, 43, 47 (Fig. 1)
Fig. 1. Ortopantomographie

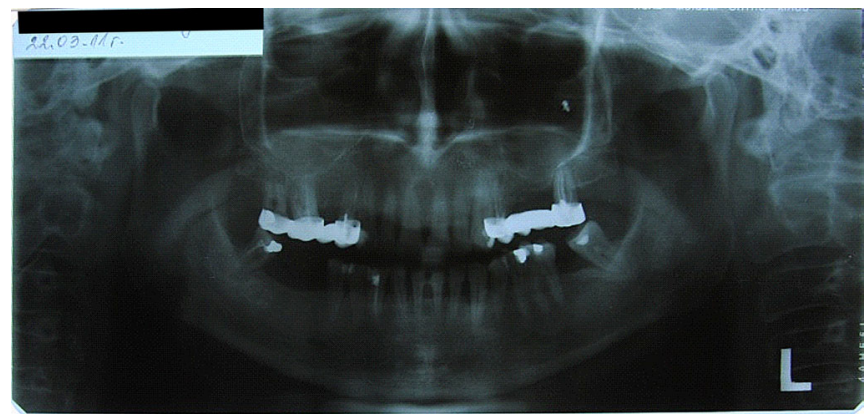

- No active dental foci were found by test of Gelen - due to the prolonged antibiotic and corticosteroid therapy

- As potential dental foci we remarked - 17, 16, 27, 43,47

- Active temperature zone - submandibular lymph nodes (Fig. 2)

Fig. 2. Thermovision research - Active temperature zone - submandibular lymph nodes

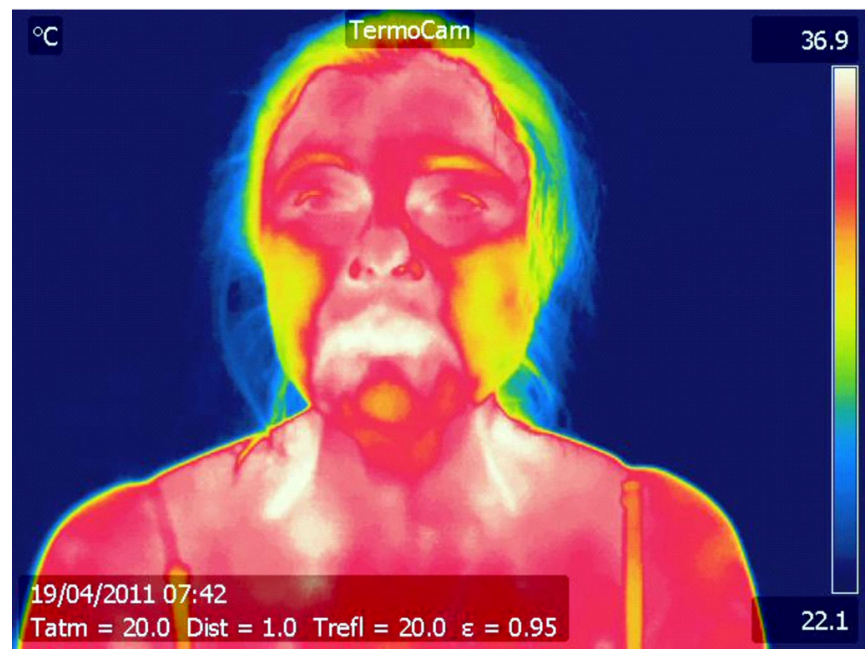

\section{DENTAL RECOMMENDATIONS}

Extraction of the potential dental foci consequently - 17, 16, 27, 43, 47 under antibiotic treatment with Clindamycin.

These focuses were extracted and the patient became afebrile 5 days afterwards last extraction. Her liver enzymes, CRP and albumin returned to normal. In addition she received Lamivudine as specific antiviral therapy for the chronic HBV-infection. 


\section{DISCUSSION}

In recent years there has been a reawakening of the dangers of oral infections and their potential disastrous effects on systemic health.

"The detrimental effect of focal infection on general health has been known for decades. Chronic dental infections may worsen the condition of medically compromised patients." (Gammal, R. 2006)

Dental sepsis is one potential cause of persistent fever that can escape detection. This diagnosis may be suggested by repeated questioning of the patient about his or her medical history, repeated physical examination, an elevated erythrocyte sedimentation rate, or a history of failure to respond to antibiotic therapy. K. Siminoski summarized that dental infection is unlikely in patients who have a white blood cell count of $>11$ Ч $109 / \mathrm{L}$, a temperature of $>39.5^{\circ} \mathrm{C}$, or positive blood cultures (Siminoski, K., 1993).

I. Karachaliou et coworkers described three patients with persistent fever due to dental disease (Karachaliou, I., 2007). L. Pernice et coworkers reported 5 cases of unexplained prolonged fever and the authors stress the need to systematically look for a dental focus of infection (Pernice, L., 1990).

Three patients with prolonged unexplained fevers were ultimately found to have deep-seated dental infection. After initial examination failed to elicit symptoms or signs of dental infection, and extensive in-hospital evaluation was nonproductive, only dental consultation with roentgenograms provided the diagnosis. All three patients underwent dental extractions with periapical or peridontal debridement; following a brief postoperative febrile period, all three responded with defervescence, without subsequent recurrence of fever (Levinson, SL. 1979).

E, Berry and J. Silver published three patients with fever and malaise, one of whom also had joint pains, were extensively investigated before their condition was attributed to dental sepsis. The authors mention that dental sepsis should be added to the list of possible causes of pyrexia of ndetermined origin, and a routine dental examination should be carried out in each case (Berry, E., Silver, J., 1976).

It is interesting that most patients with dental focus and cause of FUO have no dental symptoms, thus allowing the oral cavity to be overlooked during physical examination. Only some of these patients have complains - painful, loose teeth or discomfort with chewing, and a large percentage have abnormalities on careful oral examination, including severe periodontal disease; discolored, dead teeth; and visible or palpable abscesses (Hirschmann JV. 1997).

All this patients are needed to complete oral diagnostic procedures, including clinical examination, dental radiograph and different focal diagnostic methods.
Antimicrobial therapy in patients with fever and dental foci - yes or no

According to the necessity and need for antibiotic therapy, there are conflicting opinions, but most authors recommend that antibiotic therapy can be made before, during and after dental procedures in patients with focal dental infection.

Antimicrobial therapy alone rarely eradicates the fever and dental extraction is usually necessary (Hirschmann, JV. 1997). K. Siminoski shares that detection of dental sepsis can be cured in all instances by tooth extraction and abscess drainage, with or without concurrent antibiotic therapy.

It is important that the extraction of infected teeth leads to a cure, but, the functional disadvantages of multiple extractions need to be carefully taken into account (Pernice, L., 1990).

Any form of oral surgery will produce a bacteremia and that this may cause infections in susceptible tissues, especially the heart. What is less accepted is that other sources of sepsis exist in the mouth. These include: periodontal infections, NICO lesions (neuralgia-Inducing Cavitational Osteonecrosis), dead teeth (Gammal, R., 2006).

Dental focus of infection may not only infect other tissues but also poison the body with a variety of toxins (Gammal, R., 2006). Distribution of organisms and their toxins throughout the body is by various routes:

- blood circulation through out the body

- lymphatic distribution locally and then to blood stream

- retrograde axonal transport - transport along nerve fibres and back to the brain (Gammal R. 2006).

Based on this reports we prescribed an antibiotic protection (Clindamycin) throughout the dental extractions, which were made at several subsequent visits over a period of two weeks.

We based our recommendations on the general health of the patient, concurrent bacteremia dental extractions and the ability to avoid complications.

\section{CONCLUSION}

In a variable number of undiagnosed persistent fever cases, no definitive diagnosis is made. Oral symptoms usually are not present in patients with FUO, thus allowing the oral cavity to be overlooked during physical examination and usually fever of dental origin is neglected by the doctors. 


\section{REFERENCES:}

1. Berry E, Silver J. Pyorrhoea as cause of pyrexia. Br Med J. 1976 Nov 27; 2(6047):1289-1290. [PubMed]

2. Cunha BA. Fever of unknown origin. Infect Dis Clin North Am. 1996 Mar;10(1):111-127. [PubMed]

3. De Kleijn EM, Vandenbroucke JP, van der Meerm JW. Fever of unknown origin (FUO). I. A prospective multicenter study of 167 patients with FUO, using fixed epidemiologic entry criteria. The Netherlands FUO Study Group. Medicine [Baltimore]. 1997 Nov;76(6):392-400. [PubMed]

4. Durack DT, Street AC. Fever of unknown origin-reexamined and redefined. Curr Clin Top Infect Dis. 1991; 11:35-51. [PubMed]
5. Gammal R. THE DANGERS OF FOCAL TEETH INFECTIONS. 5th Feb 2006

6. Hirschmann JV. Fever of unknown origin in adults. Clin Infect Dis. 1997 Mar;24(3):291-300; quiz 301-2. [PubMed] [CrossRef]

7. Karachaliou I, Karachalios G, Kanakis K, Petrogiannopoulos CL, Zacharof AK. Fever of Unknown Origin due to Dental Infections: Cases Report and Review. Am J Med Sci. 2007 Feb;333(2):109-110. [PubMed]

8. Knockaert DC, Vanderschueren S, Blockmans D. Fever of unknown origin in adults: 40 years on. J Intern Med. 2003 Mar;253(3):263-275. [PubMed] [CrossRef]

9. Levinson SL, Barondess JA.
Occult dental infection as a cause of fever of obscure origin. Am J Med. 1979 Mar;66(3):463-467. [PubMed]

10. Pernice L, Ribault JY, Fourestier J, Gacon J, Quilichini R, Aubert L, et al. Persistent fever of dental origin. Rev Stomatol Chir Maxillofac. 1990;91 Suppl 1:137-8. [Article in French] [PubMed]

11. Roth A, Basello G. Approach to the Adult Patient with Fever of Unknown Origin. Am Fam Physician. 2003 Dec 1;68(11):2223-2229. [PubMed]

12. Siminoski, K. Persistent Fever Due to Occult Dental Infection: Case Report and Review. Clin Infect Dis. 1993 Apr;16 (4): 550-554. [PubMed] [CrossRef]

\section{Corresponding author:}

Assya Krasteva

Department of Oral imaging and oral diagnostic, Faculty of Dental Medicine, Medical University - Sofia 1, Sveti Georgi Sofiiski Blvd., 1000 Sofia, Bulgaria E-mail: asyakrasteva@gmail.com; 